\title{
Trivium
}

Revue franco-allemande de sciences humaines et sociales - Deutsch-französische Zeitschrift für Geistesund Sozialwissenschaften

$22^{\star} \mid 2016$

Le national-socialisme et la société allemande

\section{La décomposition de la bourgeoisie à partir de la fin du XIX siècle}

\section{Hans Mommsen}

Traducteur : Françoise Laroche

\section{(2) OpenEdition}

Journals

Édition électronique

URL : http://journals.openedition.org/trivium/5313

DOI : $10.4000 /$ trivium.5313

ISSN : 1963-1820

Éditeur

Les éditions de la Maison des sciences de l'Homme

Référence électronique

Hans Mommsen, "La décomposition de la bourgeoisie à partir de la fin du XIX siècle ", Trivium [En ligne], 22* | 2016, mis en ligne le 02 septembre 2016, consulté le 10 décembre 2020. URL : http:// journals.openedition.org/trivium/5313; DOI : https://doi.org/10.4000/trivium.5313

Ce document a été généré automatiquement le 10 décembre 2020.

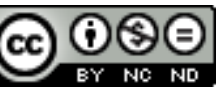

Les contenus des la revue Trivium sont mis à disposition selon les termes de la Licence Creative Commons Attribution - Pas d'Utilisation Commerciale - Pas de Modification 4.0 International. 


\title{
La décomposition de la bourgeoisie à partir de la fin du XIX ${ }^{\mathrm{e}}$ siècle
}

\author{
Hans Mommsen \\ Traduction : Françoise Laroche
}

1 Ce qui fait la teneur propre de l'époque bourgeoise, par quelles voies le concept de bourgeoisie - qui à l'origine désigne, dans la stratification féodale, la population urbaine non soumise au lien féodal - devient une catégorie idéal-typique englobant comportement social, mentalité, formes de vie et styles culturels, aucune méthode systématique ne saurait nous aider à le déterminer. Si l'on ne se contente pas de voir dans la bourgeoisie la couche intermédiaire - au sens technique - qui existe dans toutes les formations sociales, mais un phénomène socioculturel spécifique, envisager la problématique de ce phénomène à la lumière de son évolution ultérieure pourrait malgré tout avoir une fonction explicative, voire corrective. Les réflexions qui suivent représentent une telle tentative. Elles reposent sur l'hypothèse qu'à partir de la fin du $\mathrm{XIX}^{\mathrm{e}}$ siècle, les formes de vie bourgeoises se vident peu à peu de leur sens, que la bourgeoisie cultivée perd peu à peu son identité sociale, et que ces deux tendances ont pesé de façon décisive sur l'évolution politique et sociale allemande.

2 Si l'on pose l'hypothèse que la bourgeoisie a été, surtout au XIX ${ }^{e}$ siècle, aussi bien objectivement que subjectivement et par-delà toutes ses différenciations internes, une formation sociale homogène se référant à des valeurs spécifiques ${ }^{1}$, que cette homogénéité - qui se construit certes, au départ, en opposition à d'autres classes régresse au tournant du siècle, à un moment où la bourgeoisie cesse d'exister comme formation globale, on pourra peut-être dégager de ce processus de décomposition des critères qui contribueront à mieux conceptualiser notre objet. La question qui émerge alors parallèlement est celle de la durée des formes d'association politique et culturelle spécifiques à la bourgeoisie. Il en va de même des conceptions socioculturelles qui la caractérisent. Si l'on constatait alors une mutation ou une érosion des valeurs jusqu'alors reconnues, qui montrerait que la formation sociale «bourgeoisie » régresse en tant que classe, mais aussi en tant que vecteur d'une culture spécifique, cela nous 
aiderait considérablement dans notre quête de ce qui, dans la formation socioculturelle qui nous intéresse ici, se résume habituellement dans le concept de Bürgerlichkeit ${ }^{2}$.

3 L'analyse nous obligera à distinguer entre les facteurs socioéconomiques objectifs et leurs effets en retour sur l'identité et les formes d'organisation, dans la mesure où ces facteurs agissent avec un certain décalage temporel. Le bouleversement rapide de la structure sociale à la période impériale se traduit par des migrations intérieures accrues, et en particulier par l'accroissement de la population des villes, la poussée de l'urbanisation, la modification des règles de l'ascension sociale et la décomposition d'une société bourgeoise de notables jusqu'alors solidement structurée ${ }^{3}$; c'est sur cette toile de fond que s'éveille la conscience bourgeoise d'une crise qui se développe à partir du tournant du siècle ${ }^{4}$. Ce bouleversement se produit aussi bien au niveau des zones de résidence, qui commencent à perdre leur homogénéité sociale, qu'au niveau de la gestion des municipalités, où la position jusqu'alors incontestée des notables bourgeois est désormais concurrencée. Dans le même registre, la prédominance sociale des segments des couches moyennes supérieures influencés par la bourgeoisie cultivée, comme des groupes en ascension récente de la bourgeoisie économique, est menacée, notamment par l'aristocratie financière nouvelle et souvent d'origine juive. La différenciation sociale croissante des couches moyennes, qui coïncide avec la montée de la classe des employés, se traduit par un éclatement très net de la vie associative, qui représente certes des intérêts bourgeois communs face à la social-démocratie, mais poursuit aussi des buts tout à fait hétérogènes ${ }^{5}$.

Ces modifications, résumées ici à grands traits, sont perçues d'abord dans le camp bourgeois comme une crise culturelle, mais elles y suscitent dans le même temps une autocritique, qui prend à l'occasion la forme de courants antibourgeois. Le «mouvement de la jeunesse» (Jugendbewegung), qui se veut un rejet de la sécurité bourgeoise $^{6}$, les mouvements de réforme qui naissent au même moment et qu'accompagnent des phénomènes sectaires, le vitalisme et une tendance de plus en plus manifeste à l'irrationalité, tels sont les phénomènes qui traversent la bourgeoisie et qui, en tant que déviation partielle, préservent l'ensemble de la structure bourgeoise. Le marginal est toléré tant qu'il ne met pas en danger cette structure. Cela vaut pour l'acceptation des juifs assimilés comme pour les marginaux du Monte Verità, les partisans du naturisme, les anthroposophes et les autres mouvements néo-religieux qui n'ont encore fait l'objet d'aucune étude systématique ${ }^{7}$. La dénonciation de la sécurité bourgeoise - qualifiée avec mépris de "petite-bourgeoise» -, qui stigmatise une mentalité bourgeoise purement matérialiste, est typique de nombreux groupes intellectuels de la fin de siècle. Elle est étroitement liée au relativisme moral qui se répand et qui, depuis longtemps déjà, fonde la tendance à défendre des valeurs décisionnistes ${ }^{8}$.

5 La Première Guerre mondiale précipite ces mouvements qui s'exprimaient dans l'appel à un réveil de la société, prôné par les représentants actifs de la jeune génération. Le sentiment que la guerre, d'une façon ou d'une autre, avait mis fin à l'ère de la prospérité et de la sécurité bourgeoises était largement partagé. Il s'accompagnait d'un conflit poussé jusqu'au différend idéologique entre les générations de la guerre et de l'après-guerre d'une part, et de l'autre les élites dirigeantes qui vivaient toujours dans le respect des valeurs de la société wilhelminienne ${ }^{9}$. L'agitation entretenue par l'extrême droite, par exemple par Gregor Straßer et Joseph Goebbels, la polémique contre une bourgeoisie "dépassée » étaient inséparables de la critique des «vieillards 
de Weimar ${ }^{10}$ ». Un fossé infranchissable séparait également les élites essentiellement bourgeoises de Weimar de la mentalité de la jeunesse bündisch qui, tout en se détournant d'abord de toute participation à la vie politique, était pourtant en quête des nouvelles formes communautaires qui devaient constituer le fondement de la nouvelle formation sociale à laquelle ils aspiraient ${ }^{11}$. Dans le même temps, la pensée néoconservatrice, dont il ne faut pas sous-estimer l'écho dans les milieux intellectuels, voulait revenir à l'avant-1789 et abolir ainsi non seulement les formes politiques libérales, mais aussi l'individualisme bourgeois qu'elles présupposent ${ }^{12}$.

Chez les représentants des jeunes générations, être antibourgeois devint une mode sous Weimar, et il en allait de même chez les auteurs néo-conservateurs qui se trouvaient derrière le Stahlhelm, qui fournissaient des idées pour une nouvelle politique conservatrice et qui, dans les dernières années de la République, trouvèrent un remarquable porte-parole dans la revue Die Tat de Hans Zehrer ${ }^{13}$. Mais des sentiments équivalents animaient la gauche réformiste, qu'exprimait Theodor Haubach quand il parlait du cordon sanitaire établi par la République contre les forces de la jeune génération ${ }^{14}$. Ce conflit de générations dissimulait toutefois un nouveau sentiment de l'existence, qui se voulait antibourgeois alors même qu'il ne pouvait prospérer que sur le terreau des formes de vie spécifiquement bourgeoises. Les corps francs, qui se recrutaient à peu près exclusivement dans la bourgeoisie, en sont une autre illustration. Dénonçant la société bourgeoise comme décadente, ils développèrent une contre-culture héroïco-militaire issue du rejet profond d'un XIX siècle repu, et dont Ernst von Salomon fut un représentant typique ${ }^{15}$. Il fut également le chantre de la violence en politique, qui, comme Hitler eut l'intelligence de le pressentir, trouva un écho favorable dans des pans entiers de la bourgeoisie. Avec le rejet du règlement institutionnalisé et organisé des conflits, avec la fuite vers un mythe esthétisé de la violence, on touche du doigt l'abandon par la bourgeoisie de ses convictions morales ${ }^{16}$, même si cet abandon était en relation dialectique avec un attachement à des représentations spécifiquement bourgeoises de l'ordre.

7 C'est dans le champ politique qu'il est le plus facile de montrer l'érosion des formes sociales spécifiquement bourgeoises. On ne se trompera guère en considérant l'association de type Verein comme la forme d'organisation culturelle et politique typique de la bourgeoisie et en la reliant étroitement au concept d'« espace public de la bourgeoisie ${ }^{17}$ ». A la différence des formes préconstitutionnelles d'organisation politique - clubs, cercles de lecture, sociétés secrètes, salons -, qui représentent une phase de transition, la forme associative du Verein requiert par essence l'espace public, que ce soit dans son organisation interne ou dans ses liens avec l'extérieur, et par principe elle est définie par la libre adhésion des personnes partageant les mêmes convictions. Il n'est pas atypique qu'avec la consolidation du système constitutionnel et le passage à des formes parlementaires, les partis politiques aient adopté - avec, malgré tout, un certain retard dans le camp conservateur - cette forme d'association, qui fut ensuite supplantée par des formes intégratives de groupement (Verband) d'intérêts ${ }^{18}$. Cela vaut pour la plupart des groupements bourgeois, avec cependant, très tôt, des formes intermédiaires.

8 C'est donc un changement significatif qui se produit lorsqu'au tournant du siècle le Verein, forme jusqu'alors privilégiée d'organisation politique et associative, recule ou perd ses aspects spécifiquement publics. Ce mouvement, qui s'était amorcé dès le dernier tiers du XIX ${ }^{e}$ siècle, s'amplifie pendant et surtout après la Première Guerre 
mondiale. On ne sera pas étonné de le voir s'exprimer le plus puissamment à l'aile droite des groupements bourgeois. Les dénominations varient et témoignent d'une grande imprécision (ligue, ordre, société, club): Reichshammerbund, Dürerbund, Germanenorden, Thule-Gesellschaft, Juniclub, Herrenclub, Mittwochsgesellschaft, Nationaler Club von 1919. Toutes ces organisations, dont le nombre et les métamorphoses rendent vain tout essai d'inventaire, se caractérisent par un recours à des formes associatives prélibérales, qui, à la liberté d'adhésion et de recrutement, substituent les principes de la cooptation, de l'admission ritualisée des nouveaux membres et d'une existence à la marge de l'espace public. Le mouvement dit RingBewegung, qui décrit la constitution des groupes sociaux dirigeants par un mouvement de cercles recrutant autour d'eux, est sans nul doute une tendance caractéristique de la décomposition de la structure bourgeoise antérieure, même si elle est aussi présente dans les milieux de l'aristocratie et n'épargne pas non plus la gauche politique, comme le montre l'existence d'organisations telles que l'ISK ou le cercle constitué autour des Neue Blätter für den Sozialismus ${ }^{19}$.

ne saurait surestimer les conséquences politiques et sociales de ce mouvement à dominante néo-conservatrice qui marque les années 1920. Même si une grande partie des notabilités qu'il concernait jouaient simultanément un rôle dans les partis politiques, le réseau des élites qui se constituait ainsi était une organisation de recrutement, mais aussi un substitut au système des partis bourgeois alors en perte de vitesse; ce réseau, grâce à d'innombrables relations personnelles transversales, embrassait la totalité du système politique - à l'exception de la gauche qui n'y était associée que par des liens lâches et par des transfuges de droite ${ }^{20}$. Il faut y ajouter le mouvement bündisch, dont les formes d'organisation s'écartaient fondamentalement du principe associatif bourgeois et qui se référait à la fiction de la communauté fermée de pensée et de vie, ainsi que les groupements de la droite militante, des corps francs et de leurs héritiers jusqu'à ces ligues de droite presque oubliées que furent le Bund Kreuz und Adler (Ligue de la croix et de l'aigle) ${ }^{21}$ d'Eugen Kogon et d'autres regroupements à caractère clairement politique ${ }^{22}$.

Il faut considérer tous ces cercles comme une contre-scène publique antirépublicaine, qui partait ouvertement en guerre contre la domination des partis politiques, tout en servant de milice à la droite. Un nouveau front s'esquissait ainsi virtuellement, conforme à ce que Hans Zehrer appelait ardemment de ses vœux, et qui voulait substituer une unité nouvelle aux particularismes du régime des partis ${ }^{23}$. Il signifiait aussi le retour de la bourgeoisie sur une position élitiste de front, à partir de laquelle elle espérait canaliser indirectement à son profit et, à long terme, restructurer sur des bases nouvelles une "société de masse " perçue comme hostile. Pour comprendre cela, il faut regarder de plus près l'imbrication des groupements d'intérêts bourgeois, des cercles fermés que nous venons d'évoquer et des organisations de masse de type nouveau dont la mission principale était l'endoctrinement et la mobilisation des masses populaires dans l'espace extra-parlementaire.

11 Lorsque l'Alldeutscher Verband (Ligue pangermanique) fut confronté à la réalité d'une parlementarisation achevée et de la généralisation du suffrage universel égalitaire, y compris en Prusse - c'est-à-dire à une situation qui rendait impossible la poursuite de la politique, pratiquée jusqu'alors par les notables, de recours à des canaux détournés pour influer sur le système politique ainsi que sur la presse, grâce à des liens financiers -, il entreprit de constituer des organisations parallèles de type partisan, destinées à 
« capter les masses ». Le conseiller Claß résuma cette nouvelle stratégie par la formule : "Qu'avons-nous à offrir vers le bas ${ }^{24}$ ? " Pour répondre à l'objectif de démocratisation, la collaboration entre le Germanenorden et la Thule-Gesellschaft (Société Thulé) d'une part et l'Alldeutscher Verband d'autre part aboutit à la fondation du Deutschvölkischer Schutz- und Trutz-Bund (Ligue défensive et offensive du peuple allemand), qui se proposait explicitement de mobiliser les masses autour de l'antisémitisme et de les soustraire à l'influence redoutable du mouvement socialiste. Le DAP/NSDAP naquit exactement dans le même contexte, et il est révélateur qu'Anton Drexler ait été introduit par la Thule-Gesellschaft ${ }^{25}$.

On retrouve le même type d'interpénétration dans les milieux du Deutschnationaler Handlungsgehilfenverband (DHV - Association nationale allemande des employés de commerce). Il s'agissait là aussi, à l'origine, d'une organisation périphérique de l'Alldeutscher Verband et du Christlich-Soziale Partei, qui mettait en avant l'antisémitisme. Si celui-ci était au départ tourné contre les entrepreneurs juifs qui dominaient le grand commerce, cette orientation disparut avec la montée des grandes entreprises et l'extension de la catégorie des employés. Puis la pensée syndicale s'imposa, au même rythme que l'antisémitisme cessait d'agir sur les masses ${ }^{26}$. Ainsi s'explique le paradoxe qu'après 1931 le DHV soit resté parfaitement imperméable à la volonté national-socialiste de mise au pas ${ }^{27}$. Au début, le DHV réclama dans son programme l'autonomie pour ceux de ses membres qui appartenaient aux professions commerciales, mais cela se révéla une pure illusion. De façon significative, il chercha alors à compenser cette perte de statut en mettant en place sa propre organisation de formation.

La Hanseatische Verlagsanstalt est le premier exemple éminent d'une production de livres spécifiquement destinée à une confrérie de pensée, au départ celle des membres $\mathrm{du} \mathrm{DHV}^{28}$. Ce n'est pas un hasard si les principaux dirigeants du DHV, dont Max Habermann et Hans Bechly, étaient à l'origine des libraires et représentaient la couche intermédiaire entre les éléments supérieurs de la bourgeoisie cultivée et les catégories d'employés en plein essor. Le lien avec le Dürerbund (Ligue Dürer), où Eugen Diederichs et Wilhelm Stapel jouaient un rôle important, suscita la création d'une maison d'édition autonome, qui succéda à un "cercle de lecture" (Buchgemeinschaft) destiné à ses membres. Elle allait devenir le plus grand groupe éditorial de la République de Weimar après le groupe Hugenberg. Le plus remarquable est que le marché visé n'était pas à proprement parler public, mais correspondait à une communauté de pensée völkisch (national-populiste).

Ces créations virent le jour dans un contexte de transformation radicale du marché du livre, due à la baisse des coûts de fabrication, au recul des bibliothèques de prêt et à la réduction des ventes au sein de la catégorie numériquement limitée de la bourgeoisie cultivée, mais aussi au développement de la production de masse d'une littérature populaire qui échappait au réseau des librairies de la bourgeoisie cultivée. Celle-ci, présente au Börsenverein - association professionnelle de la librairie et de l'édition -, avait perdu le monopole tacite de la distribution qu'elle exerçait jusqu'alors. La concurrence instaurée sous le signe de la lutte contre «la souillure et la honte juives ", et appuyée sur les objectifs culturels nationalistes du Dürerbund, conduisit - dans le contexte de la guerre et en relation avec la création des bibliothèques du front - à l'apparition de cercles de lecture qui avaient en même temps pour fonction, grâce à l'inflation croissante et à la paralysie des libraires que liait un système de prix fixes, de 
conquérir des segments précis du marché29. L'ancienne tradition fut ainsi minée de deux façons: d'une part, on vit s'effondrer la fiction caractéristique de la structure libérale, selon laquelle la publication de livres s'adressait à un public limité aux personnes cultivées, mais non clos; d'autre part, l'expansion des bibliothèques populaires, l'intervention de groupements bourgeois dans la distribution et la garantie d'un cercle d'acheteurs assurés, associées à une orientation du contenu de la production en fonction de certains points de vue politiques et sociaux, entraînèrent une segmentation de la culture et l'abandon de toute prétention universaliste ${ }^{30}$.

15 L'intrusion de l'économie et de la politique dans le commerce de la librairie, reflet de la décomposition de la situation de monopole de la bourgeoisie cultivée sur ce terrain, mais aussi de la crise des valeurs qu'elle traverse alors, signifie en même temps que la culture est politiquement manipulée et de plus en plus entraînée dans le sillage de l'irrationalisme politique. Ce phénomène s'inscrit dans un contexte où la rationalité bourgeoise est vidée de sa substance et où éclatent les contenus culturels autrefois conçus comme un système global. Le processus de démocratisation fondamentale se traduit par la dislocation de la communauté des intérêts bourgeois. Les groupements bourgeois qui s'efforçaient de soumettre à leur influence les masses qui n'appartenaient pas à la bourgeoisie cultivée et possédante, et de les arracher à l'emprise du mouvement ouvrier socialiste, durent faire des concessions aux thèmes populistes, parmi lesquels l'antisémitisme et le nationalisme intransigeant occupaient une place centrale ${ }^{31}$.

16 Cette tendance, qui joue à plein depuis la crise de 1873, s'exprime dans la naissance du mouvement berlinois, l'apparition de partis antisémites et l'impact inhabituellement large de l'antisémitisme intellectuel dans les milieux bourgeois ${ }^{32}$. Des enquêtes récentes montrent que ces courants ne relevaient pas de la bourgeoisie cultivée traditionnelle, mais que les associations antisémites recrutaient parmi les intellectuels qui n'avaient pas réussi à accéder à la culture et à la fortune ainsi que dans la bourgeoisie urbaine et commerciale en ascension, particulièrement perméable aux courants antisémites lorsque la récession économique s'accentua après 1873 et que les influences juives dans l'économie et la vie intellectuelle furent tenues pour responsables du déclin qui s'esquissa alors $^{33}$. C'est dans ces milieux, où l'idéalisme classique n'avait que superficiellement pénétré, que les éléments pseudo-religieux d'un antisémitisme désormais raciste et d'un nationalisme allemand se réclamant des mythes germaniques se révélèrent pleinement comme une compensation idéologique au nivellement social redouté et à la fragilité effective des statuts ${ }^{34}$.

17 L'immense popularité du credo antisémite de Houston Stewart Chamberlain ne provenait pas tant de sa critique des juifs eux-mêmes que de son utilisation de l'exemple de la race juive pour déployer le mythe d'un essor national des Allemands et du dépassement des antagonismes de classes et des clivages politiques. Le recours à l'éthique de conviction, si typique de l'idéalisme allemand, se voyait en quelque sorte renouvelé, mais le contenu moral était vidé de son sens et cédait la place à un irrationalisme empreint de volontarisme, dont on trouvait l'équivalent dans les aspirations populistes du camp bourgeois dont la traduction organisationnelle la plus large était le Vaterlandspartei (Parti de la patrie) ${ }^{35}$.

18 Le désintérêt progressif qu'inspire au cours de la première moitié du siècle la culture bourgeoise influencée par le libéralisme s'exprime de la façon la plus tangible dans l'exemple des associations Wagner ${ }^{36}$. Ces associations bourgeoises créées pour financer 
le festival de Bayreuth devinrent peu à peu une communauté de pensée dirigée centralement par les éditeurs des Bayreuther Blätter et axée sur le culte de Wagner et sur l'idéologie völkisch et antisémite qui y fut associée. Les représentants de la bourgeoisie cultivée proprement dite restèrent à l'écart du mouvement, qui fut soutenu essentiellement par la bourgeoisie possédante alors en plein essor dans les grandes villes devenues centres de commerce. Des liens transversaux de nature personnelle existaient avec le mouvement antisémite, avec le mouvement pour la protection des animaux et contre la vivisection, avec le Christlich-Soziale Reichspartei, ainsi qu'avec toutes les variantes du «mouvement de réforme " (Reformbewegung) annonciatrices de la tendance qui, au tournant du siècle, émerge sous forme de sectes, cercles, ligues et sociétés privées à caractère pseudo-religieux ${ }^{37}$. Ce qui voit le jour - dans la lignée de la tradition libérale - comme un mouvement d'accomplissement intérieur des conquêtes nationales liées à la fondation de l'Empire, se mua dès les années 1870 en une tendance délibérément rétrograde, à la fois antiparlementaire et anticapitaliste, hostile aux Lumières (Aufklärung) autant qu'au rationalisme, constituant un terrain favorable au culte de Wagner et à la critique de la culture développée par Schopenhauer et Nietzsche, puis vulgarisée et déformée par leurs épigones. Le futur mouvement néoconservateur des cercles est déjà inscrit dans les associations Wagner et dans les innombrables mouvements de « réforme de la vie » (Lebensreform).

Il n'est pas aisé d'avoir une vue générale des multiples associations qui se créent à partir de la fin des années 1890 et drainent les milieux bourgeois. Leur trait commun était un engagement en faveur d'une réforme culturelle radicale, une critique de la tradition libérale issue des Lumières et une aspiration à former des communautés de pensée tantôt proches de sectes avant-gardistes, tantôt soucieuses de favoriser le renouvellement intérieur de la société en tant que mouvement de regroupement culturel, où les conceptions völkisch devenaient de plus en plus dominantes. Les communautés de pensée remplacèrent peu à peu le type de l'association bourgeoise, qu'il s'agisse du Mitgart-Bund, du «mouvement de colonisation » (Siedlungsbewegung), de la ligue de Saint-Georges fondée par Fidus, ou de l'antisémite Hammer-Bund. Le caractère fondamentalement pseudo-religieux de ces aspirations se reflète dans les regroupements en «communes" (Gemeinden) et dans des formes de sacré séculier qui s'expriment de la façon la plus spectaculaire dans les projets de temples de Fidus ${ }^{38}$.

La régression vers une nouvelle « intériorité » et les liens délibérément renoués avec la tradition romantique pouvaient prendre un caractère purement sectaire. Mais les efforts pour élargir la base populaire du mouvement de la Kulturreform étaient nombreux. Ainsi de l'idéalisme éthique dont la traduction institutionnelle fut, pour ne citer que les groupes les plus importants, la Gesellschaft für ethische Kultur (1892), le Dürerbund (1902), le Monistenbund (1906). Ferdinand Avenarius, idéologue influent du Dürerbund et éditeur de la Kunstwart, soulignait en 1903 qu'il serait indispensable de donner corps à ses intérêts en les dotant d'une forme organisée : "Mais nous aussi, nous représentons des intérêts, de puissants intérêts publics, de la plus haute importance... Organisons-nous et nous pèserons immédiatement dans la balance des décisions ${ }^{39}$. " Fort de l'adhésion indirecte de 300000 membres, le Dürerbund était une des plus grandes organisations de ce type. Bien plus que le nombre des adhérents, l'important était l'abondance des liens transversaux avec des organisations du même type, ainsi que l'activité éditoriale et culturelle déployée par le Dürerbund. Ces liens allaient jusqu'au DHV, au Wandervogel, et par la suite à la jeunesse bündisch, au Deutscher Werkbund et à quantité d'institutions de formation populaire, d'associations 
patriotiques locales et de sociétés de gymnastique. En posant comme objectif une "culture de l'expression", Avenarius voulait combattre la "massification» tant redoutée, la culture de la grande ville ${ }^{40}$ et la fascination de la consommation ; il aspirait à un retour aux valeurs culturelles nationales qui, par des moyens esthétiques, déboucherait sur l'intégration sociale des couches moyennes et d'éléments des couches inférieures.

21 Même si la bourgeoisie cultivée jouait un rôle important au sein du Dürerbund, le noyau dur de celui-ci se recrutait dans les couches moyennes en ascension, et principalement chez les enseignants du primaire et du primaire supérieur, alors que les universitaires y étaient relativement sous-représentés ${ }^{41}$. Ses membres étaient en quelque sorte l'équivalent cultivé du terrain où se recrutait le DHV. La rupture avec les associations bourgeoises "de classe" qui dataient du milieu du XIX ${ }^{e}$ siècle était évidente. Elle tenait d'une part à la capacité de recourir aux méthodes modernes de propagande et d'organisation, et d'autre part au projet de surmonter la division sociale entre capital et travail en s'adressant aux catégories moyennes inférieures et si possible à la classe ouvrière ${ }^{42}$. Tous les groupements du Reformbewegung bourgeois - qu'ils s'ouvrent à l'occultisme, qu'ils prônent la culture comme moyen d'intégration sociale, qu'ils célèbrent le naturisme, les sports collectifs, le rejet des contraintes de la mode, la vie en communautés et en colonies rurales, une réforme agraire totale dans l'esprit de Damaschke ou le «mouvement de la terre libre » (Freilandbewegung) avec Sylvio Gsell, ou enfin qu'ils s'enthousiasment pour l'invention toute nouvelle de l'hygiène raciale et pour les idées de sélection - cherchaient à créer une sous-culture spécifiquement antimoderne, s'élevaient contre la domination du grand capital dénoncé comme juif, critiquaient le progrès de l'urbanisation et la menace de la massification, qu'ils assimilaient au développement de mœurs de plus en plus brutales et à l'anéantissement des traditions culturelles nationales.

22 L'identité bourgeoise avait comporté, entre autres éléments non négligeables, le souci de se distinguer des mœurs "frustes" des classes inférieures par la culture et la prospérité matérielle. Le cas du DHV montre que cet objectif, d'abord dominant, de défense du statut social se transforma en une stratégie empreinte de populisme alliée à l'illusion de surmonter les tensions entre classes par une politique de rassemblement national afin de parvenir à une nouvelle " communauté nationale » (Volksgemeinschaft) fondée sur une communauté de pensée. Cet objectif devait être servi par un programme d'éducation populaire global, auquel s'associèrent - bien évidemment aussi pour des raisons commerciales - les éditeurs qui en étaient proches, la Hanseatische Verlagsanstalt et l'Eugen Diederichs Verlag, et auquel la Première Guerre mondiale donna un élan supplémentaire dans le registre social ${ }^{43}$. A plus d'un égard, il s'agit là d'une transposition actualisée de la fonction intégrative de la culture bourgeoise à l'époque idéaliste, mais cette fois avec une orientation délibérément conservatrice, prenant ses distances avec la tradition libérale - orientation qui fut reprise telle quelle par le mouvement néoconservateur et qui trouva son pendant dans le camp völkisch de la République de Weimar. Si la base sociale de ce mouvement est totalement bourgeoise, la critique de l'affadissement culturel et du relativisme moral constituait un abandon très net de cette identité bourgeoise qui, au XIX siècle, se reposait sur ellemême.

23 Une condition indispensable de ce processus de différenciation et de remodelage fut la déstabilisation croissante du statut de nombreux groupes au-dessous de la grande 
bourgeoisie établie, que l'inflation commença à saper elle aussi après 1918. L'impression de perdre son enracinement social traditionnel dans la « société de masse industrielle " avait été largement confortée par les événements de la révolution de Novembre. Dès avant la fin de la guerre, Friedrich Meinecke diagnostiquait un « déclin relatif du crédit de la bourgeoisie ", déclarant peu après :

«La bourgeoisie à formation académique, autrefois en guerre contre les anciennes couches dirigeantes, puis unie à celles-ci et en partie fondue en elles pour une certaine participation au pouvoir, se sent désormais sur la défensive face à ces catégories mêmes qui sont apparues avec le passage de l'État agraire à l'État industriel : les larges masses des ouvriers et des employés ${ }^{44} »$.

L'inquiétude quant au statut devint désormais un sentiment fondamental jusque dans les couches moyennes supérieures - professeurs, hauts fonctionnaires ou membres des classes moyennes industrielles, artisanales et commerciales, à présent exposés à la concurrence des grands Konzern, des grands magasins et des chaînes de discount ${ }^{45}$.

Le conflit interne à la bourgeoisie, qui coïncidait largement avec l'opposition entre anciennes et nouvelles classes moyennes, s'exprima notamment dans le milieu associatif. De façon caractéristique, le Reichsbund der höheren Beamten (Fédération des hauts fonctionnaires du Reich) se sépara du DBB et combattit avec acharnement le nivellement croissant des salaires dans la fonction publique au détriment de l'élite des fonctionnaires ${ }^{46}$. Le secrétaire d'État Hans Schäffer lui-même mit Brüning en garde contre l'irritation des hauts fonctionnaires qui n'avaient plus les moyens de se payer une femme de chambre ${ }^{47}$. Pour l'artisanat et les classes moyennes industrielles et commerciales qui se sentaient les principales victimes de la stabilisation et de la dépression, des tensions supplémentaires virent le jour à la tête des organisations centrales de la grande industrie et de l'agriculture. Larry Jones a décrit en détail l'émiettement des intérêts politiques du centre qui en résulta ${ }^{48}$. L'influence rétrograde des notables bourgeois pesait aussi sur les politiques communales. Il ne faut toutefois pas surestimer le poids des partis bourgeois. Jusqu'au début des années 1930, les très nombreux sièges remportés par des associations libres d'électeurs allaient de plus en plus à des groupes issus des nouvelles classes moyennes, et notamment au DHV. Dans le même temps, la part des entrepreneurs et des commerçants indépendants décrût vraisemblablement par rapport à celle des dirigeants salariés dans les chambres d'industrie et de commerce ainsi que dans les autres institutions représentatives des intérêts des classes moyennes. Une exploitation systématique des monographies de groupes professionnels et de branches donnerait des informations complémentaires sur ce point.

La situation économique et sociale de la population juive pour laquelle on dispose de données détaillées est un bon exemple du déclassement social croissant des éléments de la grande bourgeoisie issus des anciennes classes moyennes ${ }^{49}$. Au plus tard à la fin des années 1920, la génération montante ne pouvait plus espérer retrouver la position sociale de ses parents, ce qui nous éclaire sur l'afflux vers les professions intellectuelles et artistiques. Le sionisme et le mouvement pour la Palestine constituent ainsi également un abandon relatif - motivé par des causes psychosociologiques - du code de comportement de la classe moyenne juive qui se référait avec insistance à la bourgeoisie cultivée. On trouverait sans doute des situations analogues pour l'ensemble de la grande bourgeoisie en déclin, même s'il ne faut pas sous-estimer la persistance de l'attachement formel à un style de vie économiquement indépendant. Un indicateur en 
est l'accentuation de l'autocritique bourgeoise qui culmine chez Ernst Jünger, dont Léopold Schwarzschild attribue le nationalisme héroïque à une " haine extrême de la Bürgerlichkeit », en tant qu' « antithèse de l'héroïsme ${ }^{50}$ ». La figure de l'ouvrier dont Jünger érige la stylisation en système est l'antithèse exacte du bourgeois, dont la défense du juste milieu et de contenus culturels désormais stériles et politiquement dépourvus de fonction ne relève plus que du musée ${ }^{51}$.

Ce qui, chez Jünger et les néo-conservateurs de la même veine, apparaît comme une évasion individuelle hors du mode de vie bourgeois se retrouve sous une forme atténuée comme tendance générale d'une bourgeoisie déstabilisée dans son leadership culturel habituel. L'élément constitutif de son identité politique demeure son opposition au monde ouvrier, qui s'exacerbe de façon quasi dramatique en un antibolchevisme hypertrophiés2. Le sentiment d'être en quelque sorte dépassé et trahi s'exprime par une antipathie croissante envers la politique des partis et des intérêts, surtout lorsque celle-ci est placée sous le signe du républicanisme. Dès avant la Première Guerre mondiale, le Reformbewegung bourgeois, c'est-à-dire l'aile la plus activiste de la bourgeoisie, développa une aversion croissante à l'égard du système des partis politiques et de leurs activités parlementaires. Ce qui ne veut pas dire que le réseau ici esquissé de ligues, de grands groupements à tendance völkisch, nationaliste ou néo-conservatrice - réseau qui s'étend jusqu'au DHV -, n'était pas lié par toutes sortes de relations personnelles au système des partis bourgeois ${ }^{53}$.

C'est au plus tard à l'époque de Weimar que le centre de gravité des organisations propres à la bourgeoisie se déplaça vers l'espace extraparlementaire. Un élément caractéristique de cette tendance fut la naissance du Jungdeutscher Orden, au recrutement social et à l'identité politique nettement bourgeois ${ }^{54}$. Des emprunts au mouvement de jeunesse bündisch et aux corps francs en expliquent la forme d'organisation particulière, aux relents ataviques, qui recourait extérieurement à la terminologie du Deutscher Orden (Ordre teutonique). Son orientation républicaine, mais aussi la mise à l'écart des éléments paramilitaires distinguaient cet ordre des organisations de défense et des groupements patriotiques de la droite politique encore fortement empreintes de nationalisme wilhelminien. La tendance qui domina dans le camp bourgeois fut le passage du groupement d'opinion, à l'origine expressément conçu comme extraparlementaire, au parti politique, qui s'exprima en 1930 par la fusion - certes peu couronnée de succès - de la Volksnationale Reichsvereinigung (Union nationale-populaire du Reich) et du DDP pour former le Staatspartei (Parti de l'Etat) $)^{55}$. L'Ordre fut l'exemple le plus marquant de la pénétration du principe bündisch dans le système politique. Le mouvement des camps de travail lancé au départ par Arthur Maraun avait pour objectif la construction de la communauté nationale (Volksgemeinschaft) par la régénération interne du corps du peuple ${ }^{56}$. De même que le DHV, le Jungdeutscher Orden avait définitivement tourné le dos à la tradition politique bourgeoise du XIX ${ }^{e}$ siècle. Le retour à des modes d'organisation corporatistes, qui était typique de ces deux groupements et qui gagnait en même temps du terrain dans la presse politique de l'époque ${ }^{57}$, était synonyme de l'abandon défensif du leadership politique et social de la bourgeoisie qui passait autrefois pour évident. d'un nouvel ordre social global qui devait mettre fin à l'émiettement des intérêts et à la lutte des classes prolétariennes, et donner le jour à un ordre corporatif dans lequel la bourgeoisie pourrait reprendre sa place habituelle et sa fonction culturelle dirigeante ${ }^{58}$. 
L'idée d'un "réveil national", propagée pendant la Première Guerre mondiale et reprise d'autant plus vigoureusement après la défaite, avait été préparée idéologiquement par les mouvements de réforme bourgeois dès la fin du $\mathrm{XIX}^{\mathrm{e}}$ siècle. Face à la conscience apocalyptique d'une crise, cette idée dut paraître en quelque sorte libératrice, puisque porteuse d'unité dans le camp dispersé des regroupements d'opinion qui s'étaient produits au sein des partis bourgeois. Le mythe d'une nouvelle intégration créatrice de valeurs, souvent associé au thème du guide (Führer), pesait désormais d'un poids décisif dans le camp bourgeois, et - à part l'extrême gauche du DDP, isolée dès 1919 - cela vaut pour tous les partis bourgeois, à l'exception du Zentrum qui à partir de 1929 commença toutefois à rejoindre cette tendance ${ }^{59}$, assortie d'idées constitutionnelles autoritaires ${ }^{60}$.

31 La désagrégation accélérée du camp bourgeois sous l'effet de l'inflation galopante interdit néanmoins tout discours généralisateur. L'érosion des partis d'opinion libéraux au bénéfice, tout d'abord des partis d'intérêts, puis du NSDAP, était l'expression du désarroi croissant de l'ancienne classe moyenne ${ }^{61}$. Paradoxalement, le NSDAP, qui s'était à l'origine constitué en opposition à la bourgeoisie, se révéla le plus important lieu de rassemblement de membres de l'ancienne classe moyenne jusqu'alors mal intégrés ou déçus par le DNVP. A l'inverse, le groupe en expansion des employés ne fut initialement pas entraîné dans le sillage du NSDAP, et ne le fut que peu par la suite. Les préjugés sociaux firent reculer les notables bourgeois, mais ces préventions finirent par tomber au début des années $1930^{62}$. Dans l'implantation du NSDAP comme mouvement de masse, l'intégration des associations locales bourgeoises - en général peu politisées -, héritières de la tradition bourgeoise du XIX siècle, joua un rôle considérable ${ }^{63}$. De même, le NSDAP réussit en un tournemain à s'imposer à la place des associations libres d'électeurs dans les institutions représentatives communales. La même chose se produisit lors des élections aux assemblées protestantes de $1932^{64}$.

La percée du NSDAP à partir de 1929 fait suite à une décomposition accélérée du spectre des partis bourgeois et à un basculement à droite de l'électorat bourgeois que ne put contenir durablement le DNVP. En tant que parti protestataire, le NSDAP profita en première ligne de l'opposition de plus en plus vive entre les secteurs modernes et traditionnels de la société. Après avoir réalisé sa percée la plus forte dans le camp paysan, il parvint peu à peu à capter la fraction de la bourgeoisie frappée ou menacée de déclin social ${ }^{65}$. Il serait pourtant erroné d'en conclure à un effondrement complet des valeurs bourgeoises. Certes, Hitler et les propagandistes du NSDAP évitaient de camper sur des positions bourgeoises, même si depuis 1927 ils avaient clairement opté pour le maintien de l'ordre de la propriété capitaliste - malgré quelques fausses notes dans leur propagande. L'accent mis sur l'antibolchevisme, la reprise du mythe du réveil national et la volonté de revenir à la stabilité politique et économique répondaient cependant à la frustration d'une part importante de l'ancienne classe moyenne. Le slogan du retour à une fonction publique professionnelle témoigne ainsi de l'habileté du NSDAP à utiliser le désir des milieux bourgeois de voir garantis ou restaurés leurs privilèges antérieurs ${ }^{66}$.

L'interprétation si répandue du nazisme comme fossoyeur de la bourgeoisie - qui s'appuie sur des citations tirées en général des Conversations de Hermann Rauschning avec Hitler ${ }^{67}$ - est erronée.

La politique du NSDAP en direction de la classe moyenne fut un échec, au même titre que la colonisation rurale ${ }^{68}$. Mais on ne peut parler non plus de déstabilisation des 
positions bourgeoises et, sur le plan idéologique, le régime s'inscrivit dans la continuité des traditions nationales bourgeoises du XIXe siècle. Dans leur vie privée, et même si le fossé avec la bourgeoisie établie resta insurmontable, les satrapes imitèrent le style de représentation de la grande bourgeoisie. Le national-socialisme ne fut pas le principal responsable de l'effondrement des conceptions morales bourgeoises. En privilégiant la garantie de leur statut, les individus cédèrent à une indifférence morale croissante qui $\mathrm{y}$ contribua aussi ${ }^{69}$. Ainsi s'explique l'absence de résistance des forces bourgeoises à une pratique politique nationaliste profondément contraire à la conception bourgeoise de l'ordre; ainsi s'explique aussi leur propension à en évacuer les conséquences criminelles et à se raccrocher à la fiction d'un retour à l'ordre bourgeois réduit au principe formel d'un État de droit ${ }^{70}$. Il parait donc logique qu'après l'effondrement du Troisième Reich, ces mêmes forces bourgeoises se soient proposées comme garantes du nouvel ordre politique, à propos par exemple du droit de la fonction publique, des universités et de l'éducation, et qu'elles aient en même temps manifesté leur désir de recouvrer leur position économique antérieure.

Expliquer la prise de pouvoir national-socialiste par les habituelles formules de "panique des classes moyennes", d'« extrémisme du centre» ou de "suicide de la bourgeoisie $»^{71}$, c'est méconnaître la complexité des processus par lesquels le NSDAP s'est imposé comme mouvement de masse et a fini par être appelé au gouvernement du Reich. Il est plus raisonnable de parler de non-viabilité d'une République de Weimar abandonnée par de nombreux éléments de la bourgeoisie. Leur absence de résistance morale, qui, sous le régime nazi, s'amplifia au-delà de toute mesure, constitue néanmoins l'apogée d'une évolution amorcée bien avant la Première Guerre mondiale cet aveuglement idéologique croissant qu'a notamment étudié Fritz Stern ${ }^{72}$. Il n'est pas sûr, cependant, qu'il faille relier en première ligne cette crise - nullement unique en son genre - du système démocratique parlementaire en Allemagne à la faiblesse relative du libéralisme bourgeois, même si l'autoritarisme souvent dénoncé de la société allemande et son absence de vision pragmatique de la politique ont permis aux structures politiques fascistes de se mettre en place pratiquement sans résistance. L'opposition entre les secteurs modernes et arriérés de la société, qu'accentuèrent la défaite, l'inflation galopante, la stagnation puis la crise économique, affecta également le centre bourgeois, qui ne peut être considéré ni politiquement ni socialement comme formant une unité. Le "Tiers-État», au contraire, éclata en camps antagonistes, en lobbies divergents, en groupes professionnels poursuivant des intérêts différents ${ }^{73}$. Il est inutile d'expliquer en détail comment la majeure partie de l'ancienne classe moyenne, notamment la bourgeoisie cultivée, réagit par l'amertume et le ressentiment à la constitution du groupe social des employés et à la montée de nouveaux groupes professionnels dans le commerce, l'industrie et l'artisanat ainsi que dans les services publics et privés. Le privilège classique de la culture avait perdu de son importance antérieure dès la fin du XIX ${ }^{e}$ siècle. De nouvelles filières de formation, de nouvelles formes de transmission du savoir, mais aussi les nombreuses entreprises de vulgarisation des éléments culturels - éléments qu'avait remodelés l'idéalisme afin de se rallier les couches moyennes inférieures alors en expansion (comme le faisait par exemple le Dürerbund) --, ne firent qu'encourager la fuite, qui se généralisa dès les années 1870, vers des comportements élitistes et souvent ésotériques, dont le dénominateur commun fut le rejet des Lumières et des valeurs libérales traditionnelles. Les représentants de ce courant, qui se rassemblèrent dans le mouvement de la Lebensreform avant d'éclater en toutes sortes de sectes dans les années $1920^{74}$, n'étaient 
en général pas issus de la bourgeoisie cultivée traditionnelle, mais de groupes bourgeois récents dont le ressentiment social trouva une soupape dans le nationalisme et dans un antisémitisme raciste, et à qui l'ancienne religion idéaliste de la culture n'offrait plus de compensation suffisante à la fragilité de leur statut social. Le wagnérianisme ouvrit ce mouvement, qui propagea dans les milieux intellectuels et artistiques une vision du monde teintée d'idéalisme et de romantisme.

C'est ainsi qu'en 1932 Theodor Geiger, devant les statistiques sociales et les données sur les mentalités sociales dont il disposait, fut conduit à mettre en garde non seulement contre une utilisation non contrôlée du concept de classe moyenne, mais aussi contre une fusion de l'ancienne et de la nouvelle classe moyenne sous le concept unique de "bourgeoisie ${ }^{75}$ ». Il s'éleva notamment contre le concept - à ses yeux générateur de confusion - de Bürgerlichkeit qui, appliqué au contexte social nouveau du $\mathrm{XX}^{\mathrm{e}}$ siècle, se réduisait alors à des critères purement formels de la vie matérielle. Il réfuta avec énergie l'hypothèse selon laquelle la Bürgerlichkeit, en tant que façon de vivre et de penser, rapprochait l'une de l'autre l'ancienne et la nouvelle classe moyenne :

"Même si cela était vrai pour certains éléments des générations les plus âgées des deux groupes, même s'il s'agissait dans ce cas d'une seule et même Bürgerlichkeit et non de deux mentalités différentes réunies sous une même dénomination - même dans ce cas, cela ne pourrait être considéré que comme une survivance. La jeunesse de toutes les catégories de population a perdu toute idée et tout sens de la Bürgerlichkeit. Pour nous, représentants d'une génération plus ancienne, la Bürgerlichkeit était encore une forme de vie que nous pouvions nous représenter, même si nous la rejetions pour nousmêmes... Pour la génération la plus récente, la Bürgerlichkeit est un terme qui n'appelle même plus d'explication, qui se situe au-delà même de l'intérêt que suggère un refus. Elle n'a même plus idée que cela ait pu exister.»

Cela s'appliquait selon lui aux universitaires et aux employés, aux industriels et aux commerçants. «La jeunesse du début de ce siècle se révolta contre la génération de ses parents et contre leur mode de vie ; la jeunesse du deuxième quart du siècle sembla se contenter d'attendre avec indifférence le dépérissement de ce qui la précédait ${ }^{76}$.

De fait, à la fin de l'ère de Weimar, les bases sociales de la bourgeoisie comme formation socioculturelle relativement homogène étaient désormais des coquilles aussi vides que les valeurs et les modes de vie qui la caractérisaient. Malgré tous ses efforts, la tradition de la culture ne put imposer l'intégration, et elle s'engagea bien davantage sur le terrain glissant des aspirations völkisch et nationalistes. Le nouveau culte des « sciences de l'esprit » (Geisteswissenschaften) et l'outrecuidant détournement élitiste et politique de la tradition idéaliste en une critique globale de la prétendue « société de masse », le triomphe d'un mythe du Führer et d'une harmonie nationaliste dépassant le relativisme moral, s'inscrivent dans le même contexte. L'aspiration à une communauté nationale (Volksgemeinschaft) où régnerait l'harmonie sociale ne pouvait que difficilement se substituer à une société bourgeoise culturellement et économiquement solide. L'irrationalisme politique reflétait le sentiment de crise qui dominait dans la bourgeoisie depuis la fin du siècle. Victime de son désir de s'affirmer en tant que " classe ", la bourgeoisie devint le jouet d'une manipulation mûrement réfléchie de son ressentiment.

40 Cette évolution était sans doute inévitable. Tous les pays occidentaux semblent avoir traversé une profonde crise interne, provoquée par la décomposition de la tradition libérale et par la perte de légitimité du parlementarisme libéral. En Allemagne, elle fut 
plus précoce et plus violente qu'en France ou en Angleterre, mais bien plus tardive que dans les Etats orientaux de l'Europe centrale, ce qui lui conféra un bien plus grand pouvoir destructeur. Le développement des structures de la société industrielle l'empêcha de basculer dans la dictature autoritaire. Cela s'explique peut-être par la faiblesse de la couche bourgeoise qui, prisonnière de représentations politiques rétrogrades, devint une étrangère dans la République parlementaire et jugea vains tous les efforts de cette dernière pour prendre en compte ses intérêts. Carl Goerdeler illustre de façon exemplaire sa résistance à Hitler. La revendication, pour lui centrale, d'une restauration " de la correction et de l'ordre " (Anstand und Ordnung) était une formule bourgeoise. Dans le contexte de l'époque, elle ne pouvait - et de loin - suffire à donner une base assez large au coup d'Etat ${ }^{77}$. La tragédie de la politique bourgeoise dans la situation créée par le Troisième Reich s'exprime bien dans cet exemple.

\section{BIBLIOGRAPHIE}

Allen, W. S. (1981) : « Die deutsche Öffentlichkeit und die Reichskristallnacht - Konflikte zwischen Werthierarchie und Propaganda im Dritten Reich », in : Peukert, D. / Reulecke, J. (éd.) : Die Reihen fest geschlossen. Beiträge zur Geschichte des Alltags unterm Nationalsozialismus, Wuppertal, p. 397-412.

Aufmuth, U. (1979) : Die deutsche Wandervogelbewegung unter soziologischem Aspekt, Göttingen. Baum, R. C. (1981) : The Holocaust and the German Elite. Genocide and National Suicide in Germany 1871-1945, Ottawa.

Bennathan, E. (1966) : « Die demographische und wirtschaftliche Struktur der Juden », in : Mosse, W. E. : Entscheidungsjahr 1932. Zur Judenfrage in der Endphase der Weimarer Republik, Tübingen, p. 87-131.

Bergmann, K. (1970) : Agrarromantik und Großstadtfeindlichkeit, Meisenheim.

Boehlich, W. (1965) (éd.) : Der Berliner Antisemitismus-Streit, Francfort.

Bohrer, K. H. (1978) : Die Ästhetik des Schreckens : Die pessimistische Romantik und Ernst Jüngers Frühwerk, Munich.

Born, K. E. (1963) : « Der soziale und wirtschaftliche Strukturwandel Deutschlands am Ende des 19. Jahrhunderts ", Vierteljahrschrift für Sozial- und Wirtschaftgeschichte, 60, p. 361-376 (aussi in : Wehler, H.-U. : Das deutsche Kaiserreich 1871-1918, Göttingen, 1973, p. 30 s.).

Breuning, K. (1969) : Die Vision des Reiches. Deutscher Katholizismus zwischen Demokratie und Diktatur (1929-1934), Munich.

Bühler, K. (1979) : Die pädagogische Problematik des freiwilligen Arbeitsdienstes, thèse, Aix-laChapelle.

Childers, Th. (1983) : The Nazi Voter. The Social Foundations of Fascism in Germany, 1919-1933, Chapel Hill.

Diehl, J. M. (1977) : Paramilitary Politics in Weimar Germany, Bloomington. 
Döhn, L. (1970) : Politik und Interesse. Die Interessenstruktur der Deutschen Volkspartei, Meisenheim. Domansky, E. (1986) : Politische Dimensionen von Jugendprotest und Generationenkonflikt in Europa im 20. Jahrhundert, Bonn.

Edmundson, N. (1966) : « The Revolution », Journal of Modern History, 28, p. 160-180.

Ehni, G. / Weissbach, F. (1967) : Buchgemeinschaften in Deutschland, Hambourg.

Falter, J. W. (1982) : «Radikalisierung des Mittelstandes oder Mobilisierung der Unpolitischen? », in : Steinbach, P. (éd.) : Probleme politischer Emanzipation im Modernisierungsprozeß, Stuttgart, p. 438-469.

Falter, J. W. (1991) : Hitlers Wähler, Munich.

Fenske, H. (1973) : « Beamtenpolitik in der Weimarer Republik », Verwaltungsarchiv, vol. 64, p. 117-135.

Franz-Willing, G. (1962) : Die Hitler-Bewegung. Der Ursprung 1919-1922, Hambourg.

Frécot, J. (1976) : « Die Lebensreformbewegung », in : Vondung (1976), p. 138-152, 196 s.

Frécot, J. / Geist, J. F. / Kerbs, D. (1972) : Fidus 1868-1948. Zur ästhetischen Praxis bürgerlicher Fluchtbewegungen, Munich.

Fritzsche, K. (1976) : Politische Romantik und Gegenrevolution. Fluchtwege in der Krise der bürgerlichen Gesellschaft : Das Beispiel des Tat-Kreises, Francfort.

Fügen, H. N. (1974) : « Der George-Kreis in der "dritten Generation" », in : Rothe, W. (éd.) : Die deutsche Literatur in der Weimarer Republik, Stuttgart, p. 334-358.

Fullerton, R. A. (1977) : « Creating a Mass Book Market in Germany. The Story of the "Colporteur Novel" 1870-1890 », Journal of Social History, 10, p. 265-283.

Geiger, Th. (1967) : Die soziale Schichtung des deutschen Volkes. Soziographischer Versuch auf statistischer Grundlage, 2e éd., Stuttgart.

Gerstenberger, H. (1969) : Der Revolutionäre Konservatismus. Ein Beitrag zur Analyse des Liberalismus, Berlin.

Goebbels, J. (1926) : Die Zweite Revolution. Briefe an Zeitgenossen, Zwickau.

Greiff, W. (1985) : Das Boberhaus in Löwenberg/Schlesien 1933-1937. Selbstbehauptung einer nonkonformen Gruppe, Sigmaringen.

Habermas, J. (1962) : Strukturwandel der Öffentlichkeit. Untersuchung zu einer Kategorie der bürgerlichen Gesellschaft, 2e éd., Neuwied.

Haltern, U. (1974) : « Bürgerliche Gesellschaft - Theorie und Geschichte », Neue Politische Literatur, 19 , p. $472-488$.

Haltern, U. (1975) : « Bürgerliche Gesellschaft - Theorie und Geschichte », Neue Politische Literatur, 20, p. 45-59.

Haltern, U. (1985) : Die bürgerliche Gesellschaft, Darmstadt.

Hamel, I. (1967) : Völkischer Verband und nationale Gewerkschaft. Der Deutschnationale Handlungsgehilfenverband 1893-1933, Francfort.

Hamilton, R. F. (1982) : Who voted for Hitler?, Princeton.

Haubach, Th. (1930) : Die Generationenfrage und der Sozialismus. Soziologische Studien zur Politk, Wirtschaft und Kultur der Gegenwart. Alfred Weber gewidmet, Berlin. 
Henning, H. (1972) : Das westdeutsche Bürgertum in der Epoche der Hochindustrialisierung 1860-1914. Soziales Verhalten und soziale Strukturen, t. I : Das Bildungsbürgertum in den preußischen Westprovinzen, Wiesbaden.

Hermand, J. (1972) : Der Schein des schönen Lebens, Francfort.

Heydorn, H. J. / Koneffe, G. (1973) : Studien zur Sozialgeschichte und Philosophie der Bildung, II : Aspekte des 19. Jahrhunderts in Deutschland, Munich.

Hiller, H. / Strau, W. (1975) : Der deutsche Buchhandel, $5^{\mathrm{e}}$ éd., Hambourg.

Holzbach, H. (1981) : Das « System Hugenberg ». Die Organisation bürgerlicher Sammlungspolitik vor dem Aufstieg der NSDAP, Stuttgart.

Jamin, M. (1984a) : «Das Ende der "Machtergreifung” : Der 30. Juni 1934 und seine Wahrnehmung in der Bevölkerung », in : Michalka, W. (éd.) : Die nationalsozialistische Machtergreifung, Paderborn, p. 207-219.

Jamin, M. (1984b) : Zwischen den Klassen. Zur Sozialstruktur der SA-Führerschaft, Wuppertal.

Jesse, E. (1993) : « Hermann Rauschning, Der fragwürdige Kronzeuge », in : Smelser, R. L. et al. (éd.) : Die braune Elite, vol. 2, Darmstadt, p. 193-205.

Jones, L. E. (1972) : «"The Dying Middle" : Weimar Germany and the Fragmentation of the Bourgeois Politics ", Central European History, 5, p. 23-54.

Jones, L. E. (1976) : « Between the Fronts : The German National Union of Commercial Employees from 1928 to 1933 », Journal of Modern History, 48, p. 462-482.

Jones, L. E. (1977) : « Sammlung oder Zersplitterung. Die Bestrebungen zur Bildung einer neuen Mittelpartei in der Endphase der Weimarer Republik », Vierteljahreshefte für Zeitgeschichte, 25 (3), p. 265-304.

Jones, L. E. (1988) : German Liberalism and the Dissolution of the Weimar Party System, 1918-1933, Chapel Hill.

Karl, W. (1973) : Jugend, Gesellschaft und Politik im Zeitraum des Ersten Weltkrieges, Munich.

Kater, M. H. (1983) : The Nazi Party. A Social Profile of Members and Leaders 1919-1945, Cambridge/ Mass.

Kessler, A. (1976) : Der Jungdeutsche Orden in den Jahren der Entscheidung, vol. 2 : 1931-1933, Munich.

Kessler, H. (1967) : Wilhelm Stapel als Publizist, Nuremberg.

Kocka, J. (1983) : Bürger, Bürgerlichkeit und bürgerliche Gesellschaft, ms., Bielefeld.

Kocka, J. (1987) (éd.) : Bürger und Bürgerlichkeit im 19. Jahrhundert, Göttingen.

Koebner, Th. (1982) : « Die Erwartung der Katastrophe. Zur Geschichtsprophetie des "neuen Konservatismus" ", in : id. (éd.) : Weimars Ende. Prognosen und Diagnosen in der deutschen Literatur und politischen Publizistik (1930-1933), Francfort, p. 348-359.

Kornhass, E.-W. (1976) : « Zwischen Kulturkritik und Machtverherrlichung : Kurt Riezler », in : Vondung (1976), p. 92-105.

Koshar, R. (1982) : « Two "Nazisms" : the Social Context of Nazi Mobilization in Marburg and Tübingen », Social History, 7, p. 27-42.

Koshar, R. (1985) : Organization Life and Nazism : A Study of Mobilization in Marburg an der Lahn, University of Michigan Press. 
Krabbe, W. (1974) : Gesellschaftsveränderung durch Lebensreform. Strukturmerkmale einer sozialreformerischen Bewegung im Deutschland der Industrialisierungsperiode, Göttingen.

Kratzsch, G. (1969) : Kunstwart und Dürerbund. Ein Beitrag zur Geschichte der Gebildeten im Zeitalter des Imperialismus, Göttingen.

Kunz, A. (1982) : «Stand versus Klasse, Beamtenschaft und Gewerkschaften im Konflikt um den Personalabbau 1923/1924 », in : Geschichte und Gesellschaft, 8, p. 55-86.

Laqueur, W. Z. (1962) : Young Germany. A History of the German Youth Movement, Londres.

Lebovics, H. (1969) : Social Conservatism and the Middle Classes in Germany 1914-1933, Princeton.

Lewy, R. S. (1975) : The Downfall of the Antisemitic Political Parties in Imperial Germany, New Haven.

Link, W. (1964) : Die Geschichte des Internationalen Jugend-Bundes und des Internationalen Sozialistischen Kampfbundes (ISK). Ein Beitrag zur Geschichte der Arbeiterbewegung in der Weimarer Republik und im Dritten Reich, Meisenheim.

Linse, U. (1983) : Barfüßige Propheten. Erlöser der Zwanziger Jahre, Berlin.

Lohalm, U. (1970) : Völkischer Radikalismus. Die Geschichte des Deutschvölkischen Schutz- und TrutzBundes 1919-1923, Hambourg.

Lokatis, S. (1993) : Hanseatische Verlagsanstalt. Politisches Buch-Marketing im « Dritten Reich », Francfort.

Lübbe, H. (1963) : Politische Philosophie in Deutschland. Studien zu ihrer Geschichte, Bâle.

Martiny, M. (1977) : «Die Entstehung und politische Bedeutung der Neuen Blätter für den Sozialismus und ihres Freundeskreises », Vierteljahreshefte für Zeitgeschichte, 25 (3), p. 373-419.

Martiny, M. (1978) : « Sozialdemokratie und junge Generation am Ende der Weimarer Republik », in : Luthardt, W. (éd.) : Sozialdemokratische Arbeiterbewegung und Weimarer Republik. Materialien zur gesellschaftlichen Entwicklung, vol. 2, Francfort, p. 56-117.

Massing, P. W. (1959) : Vorgeschichte des politischen Antisemitismus, Francfort.

Meinecke, F. (1958) : Politische Schriften und Reden (= Werke, vol. II), Darmstadt.

Merkl, P. H. (1982) : « Formen der nationalsozialistischen Gewaltanwendung : Die SA der Jahre 1925-1933 », in : Mommsen W. J. / Hirschfeld, G. (éd.) : Sozialprotest, Gewalt, Terror. Gewaltanwendung durch politische und gesellschaftliche Randgruppen im 19. und 20. Jahrhundert, Stuttgart, p. 422-440.

Mommsen, H. (1966a) : Beamtentum im Dritten Reich. Mit ausgewählten Quellen zur nationalsozialistischen Beamtenpolitik, Stuttgart.

Mommsen, H. (1966b) : « Zur Frage des Einflusses deutscher Juden auf die deutsche Wirtschaft in der Zeit der Weimarer Republik », in : Gutachten des Instituts für Zeitgeschichte, vol. 2, Stuttgart, p. 348-369.

Mommsen, H. (1973) : « Die Stellung der Beamtenschaft in Reich, Ländern und Gemeinden in der Ära Brüning ", Vierteljahrshefte für Zeitgeschichte, 21 (2), p. 151-165.

Mommsen, H. (1983) : « Zur Verschränkung traditioneller und faschistischer Führungsgruppen in Deutschland beim Übergang von der Bewegungs- zur Systemphase, in : Schieder, W. (éd.) : Faschismus als soziale Bewegung. Deutschland und Italien im Vergleich, Göttingen, p. 157-181. 
Mommsen, H. (1984) : « Die nationalsozialistische Machtergreifung und die deutsche Gesellschaft », in : Michalka, W. (éd.) : Die nationalsozialistische Machtergreifung, Paderborn, p. 29-46.

Mommsen, H. (1985a) : «Generationskonflikt und Jugendrevolte in der Weimarer Republik », in : Koebner, Th. / Janz, R.-P. / Trommler, F. (éd.) : « Mit uns zieht die neue Zeit ». Der Mythos Jugend, Francfort, p. 50-67.

Mommsen, H. (1985b) : « Der Widerstand gegen Hitler und die Deutsche Gesellschaft », in : Schmädecke, J. / Steinbach, P. (éd.) : Der Widerstand gegen den Nationalsozialismus, Munich, p. 3-23. Mommsen, W. J. (1979) : «Wandlungen der liberalen Idee im Zeitalter des Imperialismus », in : id. : Der europäische Imperialismus, Göttingen, p. 167-205.

Monte Verità, Berg der Wahrheit. Lokale Anthropologie als Beitrag zur Wiederentdeckung einer neuzeitlichen sakralen Topographie, Ausstellung der Akademie der Künste Berlin, 25. März bis 6. Mai 1979, Milan, s. d.

Morsey, R. (1977) : Der Untergang des politischen Katholizismus, Stuttgart.

Mosse, W. E. / Paucker, A. (1976) (éd.) : Juden im wilhelminischen Deutschland : 1890-1914, Tübingen. Mueller, J. (1971) : Die deutsche Jugendbewegung als Hauptrichtung neokonservatischer Reform, Zurich. Nipperdey, Th. (1961) : Die Organisation der deutschen Parteien vor 1918, Düsseldorf.

Nipperdey, Th. (1970) : «Interessenverbände und Parteien in Deutschland vor dem Ersten Weltkrieg ", in : Wehler, H.-U. : Moderne Deutsche Sozialgeschichte, Cologne, p. 369-388.

Petzold, J. (1978) : Wegbereiter des deutschen Faschismus. Die Jungkonservativen in der Weimarer Republik, Cologne.

Phelps, R. H. (1961) : « Anton Drexler - Der Gründer der NSDAP », Deutsche Rundschau, 87, p. 1134-1143.

Plum, G. (1972) : Gesellschaftsstruktur und politisches Bewußtsein in einer katholischen Region 1928-1933. Untersuchung am Beispiel des Regierungsbezirks Aachen, Stuttgart.

Pridham, G. (1973) : The Nazi Movement in Bavaria 1923-1935, New York.

Puhle, H. J. (1975) : « Parteien und Interessenverbände 1890-1914 », in : Stürmer, M. (éd.) : Das kaiserliche Deutschland. Gesellschaft und Politik 1871-1918, 2e éd., Darmstadt, p. 340-373.

Pulzer, P. (1966) : Die Entstehung des politischen Antisemitismus in Deutschland und Österreich 1867-1914, Gütersloh.

Raabe, F. (1961) : Die Bündische Jugend. Ein Beitrag zur Geschichte der Weimarer Republik, Stuttgart.

Riedel, M. (1972) : «Bürger, Staatsbürger, Bürgertum », in : Brunner, O. / Koselleck, R. / Conze, W. (éd.) : Geschichtliche Grundbegriffe, vol. 1, Stuttgart, p. 672-725.

Ringer, F. (1969) : The Decline of the German Mandarins, Cambridge/Mass.

Ritter, G. A. / Kocka, J. (1982) (éd.) : Deutsche Sozialgeschichte, II : 1870-1914, $3^{\mathrm{e}}$ éd., Munich.

Ruegg, W. / Neuloh, O. (1971) (éd.) : Zur soziologischen Theorie und Analyse des 19. Jahrhunderts, Göttingen.

Saldern, A. von (1979) : Mittelstand im Dritten Reich. Handwerker, Einzelhändler, Bauern, Francfort. Schieder, Th. (1972) : Hermann Rauschnings « Gespräche mit Hitler » als Geschichtsquelle, Opladen. 
Schneller, M. (1977) : Zwischen Romantik und Faschismus. Der Beitrag Othmar Spanns zum Konservativismus in der Weimarer Republik, Stuttgart.

Schoenbaum, D. (1980) : Die braune Revolution. Eine Sozialgeschichte des dritten Reichs, nouv. éd., Cologne.

Scholder, K. (1977) : Die Kirchen und das dritte Reich, vol. 1 : Vorgeschichte und Zeit der Illusionen, Francfort.

Schwarz, H. P. (1967) : Der konservative Anarchist. Politik und Zeitkritik Ernst Jüngers, Fribourg. Schwarzschild, L. (1966) : « Heroismus aus Langeweile », in : id. : Die letzten Jahre vor Hitler, éd. par V. Schwarzschild, Hambourg, p. 31-39.

Siegfried, K.-J. (1974) : Universalismus and Faschismus. Das Gesellschaftsbild Othmar Spanns. Zur politischen Funktion seiner Gesellschaftslehre und Ständestaatskonzeption, Vienne.

Sontheimer, K. (1959) : « Der Tat-Kreis », Vierteljahreshefte für Zeitgeschichte, 7 (3), p. 229-260.

Speier, H. (1977) : Die Angestellten vor dem Nationalsozialismus, Göttingen.

Stachura, P. D. (1981) : The German Youth-Movement 1900-1949, Londres.

Stambolis, B. (1982) : Der Mythos der jungen Generation. Ein Beitrag zur politischen Kultur der Weimarer Republik, thèse, Bochum.

Stark, G. D. (1981) : Entrepreneurs of Ideology. Neoconservative Publishers in Germany, 1890-1933, Chapel Hill.

Stearns, P. (1979) : « The Middle Class : Toward a Precise Definition », Comparative Studies in Society and History, 21, p. 377-396.

Stegmann, D. (1970) : Die Erben Bismarcks. Parteien und Verbände in der Spätphase des wilhelminischen Deutschland, Cologne.

Stegmann, D. (1972) : « Zwischen Repression und Manipulation : Konservative Machteliten und Arbeiter- und Angestelltenbewegung 1910-1918 », Archiv für Sozialgeschichte, 12, p. 351-432.

Stern, F. (1969) : Kulturpessimismus als politische Gefahr. Eine Analyse nationaler Ideologie in Deutschland, Stuttgart.

Stern, F. (1974a) : «Die politischen Folgen des unpolitischen Deutschen », in : id. : Das Scheitern illiberaler Politik. Studien zur politischen Kultur Deutschlands im 19. und 20. Jahrhundert, Francfort, p. 41-61.

Stern, F. (1974b) : « Der Zusammenbruch von Weimar », in : id. : Das Scheitern illiberaler Politik. Studien zur politischen Kultur Deutschlands im 19. und 20. Jahrhundert, Francfort, p. 212-228.

Straßer, G. (1932) : « Macht Platz, ihr Alten ! ", in: id. : Kampf um Deutschland. Reden und Aufsätze eines Nationalsozialisten, Munich, p. 171-174.

Struve, W. (1973) : Elites Against Democracy. Leadership Ideals in Bourgeois Political Thought in Germany 1890-1933, Princeton.

Tal, U. (1977) : Christians and Jews in Germany. Religion, Politics and Ideology in the Second Reich 1870-1914, Ithaca.

Trommler, F. (éd.) : « Mit uns zieht die neue Zeit ». Der Mythos Jugend, Francfort.

Tyrell, A. (1969) (éd.) : Führer befiehl. Selbstzeugnisse aus der Kampfzeit der NSDAP, Düsseldorf. 
Veltzke, V. (1987) : Vom Patron zum Paladin. Wagnervereinigungen im Kaiserreich von der Reichsgründung bis zur Jahrhundertwende, Bochum.

Vierhaus, R. (1981) (éd.) : Bürger und Bürgerlichkeit im Zeitalter der Aufklärung, Heidelberg.

Volkov, S. (1974) : « The Social and Political Function of the Late 19th Century Antisemitism : The Case of the Small Handicraft Masters », in : Wehler, H. U. (éd.) : Sozialgeschichte Heute, Göttingen, p. 416-431.

Volkov, S. (1978) : « Antisemitism as a Cultural Code. Reflections on the History and Historiography of Antisemitism in Imperial Germany », Leo Baeck Institute Yearbook, 13, p. 25-46.

Volkov, S. (1986) : « Kontinuität und Diskontinuität im deutschen Antisemitismus », Vierteljahreshefte für Zeitgeschichte, 33 (2), p. 221-243.

Vondung, K. (1976) : « Zur Lage der Gebildeten in der wilhelminischen Zeit », in : id. (éd.) : Das wilhelminische Bildungsbürgertum, Göttingen, p. 20-33, 175 s.

Wilhelm, Th. (1963) : « Der geschichtliche Ort der deutschen Jugendbewegung », in : Kindt, W. (éd.) : Grundschriften der deutschen Jugendbewegung, Cologne.

Winkler, H. A. (1972) : Mittelstand, Demokratie und Nationalsozialismus. Die politische Entwicklung von Handwerk und Kleinhandel in der Weimarer Republik, Cologne.

Winkler, H.-A. (1971) : « Der rückversicherte Mittelstand : Die Interessenverbände von Handwerk und Kleinhandel im deutschen Kaiserreich », in : Ruegg / Neuloh (1971), p. 163-179.

Wohl, R. (1979) : The Generation of 1914, Cambridge/Mass.

Wortmannn, K. (1926) : Geschichte der Deutschen Vaterlandspartei, thèse, Halle.

Zehrer, H. (1928) : « Ein Vorschlag der Verbände », Die Tat, 20, p. 123-128.

\section{NOTES}

1. Cf., dans une littérature pratiquement impossible à connaître complètement, Haltern (1974), (1975), et aussi Haltern (1985) ; Riedel (1972) ; Stearns (1979).

2. Cf. J. Kocka, in : Ritter / Kocka (1982), p. 62 s. ; Vierhaus (1981) ; cf. aussi le projet de Kocka (1983). Le concept de Bürgerlichkeit, vu sa quasi-intraduisibilité et les connotations contradictoires qu'il véhicule, apparaît comme insuffisamment opératoire. La remarque de Jürgen Kocka, à la suite de Stephan Strasser, comme quoi « la Bürgerlichkeit a marqué l'habitus et le climat social de sociétés entières, au-delà de son contexte de classe originel » - ce qui dérive du principe de l'égalité juridique, du principe de la rationalité et du discours libéral - semble amalgamer des niveaux très différents et assimiler de façon fort peu convaincante libéralisme et bourgeoisie. Cf. Kocka (1987), p. 16.

3. On ne peut ici qu'évoquer le contexte. Cf. Born (1963); W. J. Mommsen (1979), p. 178 s. On manque d'études empiriques globales sur l'Allemagne; cf. malgré tout Henning (1972).

4. Sur ce point, Stern (1969), p. 20 s. ; Vondung (1976).

5. Cf. Stegmann (1970); Puhle (1975); Nipperdey (1970); et aussi Ruegg/ Neuloh (1971) (notamment les contributions de Kaelble, Puhle et Winkler).

6. Cf. principalement Aufmuth (1979); Mueller (1971); Stachura (1981).

7. Cf. Mosse / Paucker (1976) ; Monte Verità, Berg der Wahrheit ; Frécot / Geist / Kerbs (1972) ; et aussi Hermand (1972), p. 55-127 ; Lübbe (1963), p. 142 s.

8. Cf. Lübbe (1963), p. 226 s. 
9. Cf. H. Mommsen (1985a), p. 55 s.; Karl (1973), p. 156 s.; Wohl (1979); Domansky (1986), p. 113-138.

10. Straßer (1932) et Goebbels (1926), p. 5 s.

11. Cf. Stambolis (1982), p. 27 s. ; Wilhelm (1963), p. 50 s. ; cf. aussi Laqueur (1962).

12. Lebovics (1969), p. 164 s. ; Struve (1973), p. 253 s. ; Petzold (1978), p. 211 s.

13. Fritzsche (1976) ; Sontheimer (1959), p. 229.

14. Haubach (1930); cf. Martiny (1978), p. 56 s.

15. Cf. Diehl (1977) ; Bohrer (1978).

16. Cf. Merkl (1982), p. $430 \mathrm{~s}$.

17. L'ouvrage fondamental est Habermas (1962), notamment p. $58 \mathrm{~s}$.

18. Cf. Nipperdey (1961).

19. Sur le cercle de George qui préfigure cette structure, cf. Lebovics (1969), p. 80 s.; Fügen (1974) ; Sur l'ISK, cf. Link (1964), p. 103 s. ; sur les Neue Blätter, cf. Martiny (1977), p. 373 s.

20. On ne dispose toujours pas d'une analyse systématique. Le mode de fonctionnement des relations personnelles transversales ressort très clairement de l'analyse de Holzbach (1981), p. $138 \mathrm{~s}$. et $192 \mathrm{~s}$. Cf. aussi la description, certes centrée principalement sur les «Jungkonservativen », de Petzold (1978), p. III s.

21. Breuning (1969), p. $225 \mathrm{~s}$.

22. Cf. Raabe (1961) ; sur le mouvement des corps francs, synthèse dans Diehl (1977).

23. Zehrer (1928); cf. Mommsen (1985a), p. 59 s.

24. Lohalm (1970), p. 53.

25. Cf. Franz-Willing (1962), p. 62 s. ; Tyrell (1969), p. 20 s. ; Phelps (1961), p. 1134 s.

26. Hamel (1967); cf. Jones (1976).

27. Cf. Childers (1983), p. 233 s.

28. Cf. Stark (1981), p. 22 s.

29. Cf. Stark (1981), p. 140 s. ; Kratzsch (1969), p. 369 s. Cf. également Ehni / Weissbach, (1967) ; Fullerton (1977) ; Hiller / Strau (1975).

30. Cf. aussi Heydorn / Koneffe (1973), p. 179 s.

31. Sur Stapel, cf. Gerstenberger (1969), p. 59 s. ; Kessler, H. (1967) ; Lokatis (1993).

32. Cf. Pulzer (1966) ; Massing (1959); Lewy (1975). Cf. aussi Boehlich (1965).

33. Cf. Stern (1969), p. 88 s. ; Volkov (1974) et aussi (1986), p. 231 s.

34. Cf. Volkov (1986), p. 227 s. ; également Tal (1977) ; cf. aussi Volkov (1978).

35. Cf. Holzbach (1981), p. 61 s. et aussi Wortmannn (1926) ; cf. aussi Stegmann (1970) et (1972).

36. Veltzke (1987).

37. Cf. Krabbe (1974) ; cf. Kratzsch (1969), p. 30 s., 36 s. ; Frécot (1976) ; Edmundson (1966).

38. Frécot (1972), p. 232 s., 246 s.

39. Kratzsch (1969), p. 28, 151, 336, 362.

40. Kratzsch (1969), p. 151 s. ; cf. Bergmann (1970).

41. Kratzsch (1969), p. 337 s.

42. Cf. Hamel (1967), p. 57 s.

43. Kratzsch (1969), p. 370 s.

44. Meinecke (1958), p. 388. Cf. Kornhass (1976) ; Ringer (1969).

45. Stern (1974a), p. 52 s. ; Winkler (1971).

46. Cf. Fenske (1973) ; Kunz (1982). Cf. aussi Döhn (1970), p. 272 s.

47. Cf. Mommsen (1973), p. 160 s.

48. Jones (1972), (1988).

49. Cf. Mommsen (1966b), p. 366 s. ; Bennathan (1966).

50. Schwarzschild (1966), p. 35 ; cf. Schwarz (1967), p. 71 s.

51. Schwarzschild (1966), p. 88 s. ; cf. aussi Koebner (1982), p. 354 s.

52. On ne dispose pas à ce jour d'une étude systématique. Cf. par exemple Plum (1972), p. 154 s. 
53. Cf. Mommsen (1984), p. $32 \mathrm{~s}$.

54. Cf. Kessler (1976), p. 32 s.

55. Cf. Jones (1977).

56. Sur le mouvement des camps de travail, cf. Bühler (1979) ; cf. aussi Greiff (1985), p. 17 s.

57. Sur ce sujet, voir surtout Siegfried (1974); et aussi Schneller (1977).

58. Sur le Jungdeutscher Orden, cf. Kessler (1976).

59. Sur le parti du Zentrum, cf. Morsey (1977).

60. Cf. Mommsen (1984), p. $38 \mathrm{~s}$.

61. Cf. Childers (1983), p. 228 s., 234 s. ; analyse divergente chez Falter (1982), p. 438 s.

62. Cf. Kater (1983), p. 62 s.; Hamilton (1982), p. 413 s.; Pridham (1973), p. 144 s. Récemment Falter (1991).

63. Cf. Koshar (1982), (1985).

64. Cf. Scholder (1977), p. 248 s., 255 s.

65. Cf., outre la littérature citée dans les notes 61 et 62, Mommsen (1983) et aussi Jamin (1984), p. $372 \mathrm{~s}$.

66. Cf. Mommsen (1966a), p. $46 \mathrm{~s}$.

67. Cf. Schieder (1972), p. 47 ; cf. Jesse (1993).

68. Cf. le survol qu'en propose Schoenbaum (1980), p. 170 s., $222 \mathrm{s.}$

69. Cf. Baum (1981), p. 24 s., 66 s. ; cf. p. 99 s.

70. La réaction au 30 juin 1934 est révélatrice (cf. Jamin [1984a]); de même, la réaction aux pogromes des 9-10 novembre 1938 (cf. Allen [1981]).

71. Cf. Winkler (1972), p. 31 s., 157 s. ; Saldern (1979), p. 13 s., 26 s.

72. Stern (1969), p. 341 s. ; Stern (1974b), p. 226 s.

73. Cf. Geiger (1967), p. 124 s., 131 s. Cf. aussi Speier (1977), p. 102 s.

74. Cf. Linse (1983), notamment p. 234 s.

75. Geiger (1967), p. 100, 131.

76. Geiger (1967), p. 131.

77. Cf. Mommsen (1985b), p. 15 s.

INDEX

Mots-clés : bourgeoisie, Bürgerlichkeit, communauté nationale

Schlüsselwörter : Bürgertum, Bürgerlichkeit, Volksgemeinschaft

\section{AUTEURS}

\section{HANS MOMMSEN}

Hans Mommsen (1930-2015) est considéré comme l'historien allemand le plus important du régime national-socialiste. Pour plus d'informations, voir la notice suivante. 